\title{
Violences publiques envers les
journalistes et les médias
Introduction
}

\author{
Florence Le Cam \\ Professeure \\ LaPIJ - ReSIC \\ Université libre de Bruxelles \\ Belgique \\ Florence.Le.Cam@ulb.be
}

Fábio Henrique Pereira

Professor Associado

Universidade de Brasilia

Brasil

fabiop@gmail.com

Denis Ruellan

Professeur

GRIPIC-CELSA

Sorbonne Université

France

denis.ruellan@sorbonne-universite.fr

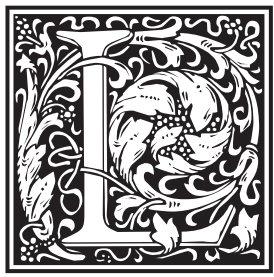

es organisations internationales de journalistes comme la Fédération internationale des journalistes (FIJ) ou Reporters sans frontières (RSF) le constatent: les violences envers les journalistes ne cessent de croître dans le monde. Anthony Bellanger de la FIJ et Maria José Braga de la Fédération nationale des journalistes au Brésil, en témoignent dans les prochaines pages de ce numéro. Des journalistes sont tué.e.s, enlevé.e.s, blessé.e.s, menacé.e.s, agressé.e.s, intimidé.e.s, harcelé.e.s, insulté.e.s. Pour leur travail, pour leurs enquêtes, pour leur appartenance à une élite, pour leur idéologie politique ou tout simplement pour être journaliste, représentant.e des journalopes ou des merdias.

La violence envers les journalistes n'est pas nouvelle. Elle prend des formes multiples depuis des décennies: critiques acerbes du métier, attaque et destruction de sièges d'entreprises médiatiques, journalistes considéré.e.s comme monnaie d'échange ou moyens de pression dans des contextes de guerre, de tensions politiques, économiques ou mafieuses. Les journalistes de guerre en sont les victimes souvent les plus médiatiques (Tumber, 2006), mais ne doivent pas masquer l'ensemble des violences subies par les journalistes locaux sur les terrains de conflits (Palmer, 2019), ou lors d'enquêtes sur les mondes fermés de la mafia, de la drogue ou des activités économiques ou politiques illicites. Ces violences publiques sont listées, répertoriées, dénoncées. Elles sont aussi l'objet de nombreux travaux de recherche qui ana-

Pour citer cet article, to quote this article, para citar este artigo :

Florence Le Cam, Fábio Henrique Pereira, Denis Ruellan, «Violences publiques envers les journalistes et les médias », Sur le journalisme, About journalism, Sobre jornalismo [En ligne, online], Vol 10, n 1 - 2021, 15 juin - june 15 - 15 de junho.

URL : http://www.surlejournalisme.com/rev 
lysent divers niveaux de violence au Moyen-Orient, en amérique du nord (González de Bustamante \& Relly, 2016) et centrale (Benítez, 2017) ou encore en Afrique (Frère, 2015). Le métier est à risque, et les violences envers les journalistes se déploient dans des dimensions diverses (Brambila \& Hughes, 2019). Certaines d'entre elles vont à l'encontre de la liberté de la presse et de la liberté d'expression (Badran, 2017), et sont devenues au fil du temps un enjeu global touchant aux droits humains (Relly \& González de Bustamante, 2017). D'autres formes de violence mettent au jour des différences genrées (Sreberny, 2014) et raciales (Louback, 2018) dans l'administration et la représentation des violences et sont aussi et surtout le reflet d'un contexte politique et idéologique qui les favorise ou à tout le moins qui ne les empêche pas.

Nerone (2008) insiste sur une série de facteurs qui peuvent expliquer ces violences : son importance dans la société ou le système politique (les normes culturelles), le niveau de professionnalisation des médias d'information et des journalistes (les normes professionnelles), et la façon dont les actions violentes sont utiles et utilisées pour marquer l'opinion publique. Ces violences incluent toujours, selon lui, une dimension symbolique forte, qui peut même être centrale pour ceux et celles qui la prodiguent. Ces dangers de l'exercice du métier sont d'ailleurs souvent abordés au travers de deux prismes: d'une part, en amont, par des réflexions sur la sécurité des journalistes dans certaines zones territoriales (pour le monde arabe, par exemple, voir Hard, 2019), dans l'étude des mesures à réaliser pour évaluer les risques (Torsner, 2017) ou encore dans la manière d'enseigner des règles de sécurité aux jeunes journalistes en formation (voir le travail sur le Pakistan de Jamil, 2018) ; d'autre part, en aval, par la dénonciation publique des violences faites aux journalistes et par des opérations de solidarité collective internationale qui tendent, comme le projet Forbidden Stories ${ }^{1}$, à poursuivre les enquêtes de journalistes tué.e.s sur le terrain et qui sont donc réduit.e.s au silence (Konow-Lund \& Olsson, 2020).

En interne, dans les rédactions et les groupes professionnels de journalistes, la question des violences est aussi un objet d'attention important, puisqu'elle permet une certaine réflexivité sur les conditions de travail et d'exercice du métier et sur la perception que les journalistes ont, collectivement, de leur autonomie (González de Bustamante \& Relly, 2016b). Et surtout de l'image publique qu'ils et elles portent, véhiculent, construisent collectivement. Ainsi, les violences subies par des journalistes lors de rassemblements publics les interrogent frontalement sur la représentation que les publics se font leur travail. Le mouvement des Gilets jaunes en France a, par exemple, rendu très claires les critiques des publics (Sebbah et al, 2018), et provoqué des réflexions sur les pratiques professionnelles et les traces qu'elles laissent dans la manière dont les publics se représentent l'écosystème médiatique et le travail de l'information (Joux, 2019). L'agressivité de certains publics envers les journalistes est ainsi relativement répandue. Fang (2017) l'explique d'ailleurs pour la Chine en prenant l'exemple de la couverture médiatique des questions de santé et insiste sur le fait qu'au-delà des modes étatiques de répression subie par les journalistes, ils et elles doivent aussi gérer les pressions et les menaces des sources comme des publics qui ont parfois tendance à faire porter la responsabilité des problèmes sociaux rencontrés sur les journalistes. Dans les contextes de forte polarisation politique, comme c'était le cas, par exemple, lors des dernières élections présidentielles au Brésil, certains journalistes sont victimes de violence numérique de la part des militant.e.s et des partis politiques au travers de l'utilisation des bots et des faux profils sur les réseaux sociaux numériques (Ramos \& Saad, 2020).

Cet ensemble de violences s'est largement déployé ces dernières années et déplacé en ligne. La première manifestation de ces violences renvoie, dans certains pays, à la surveillance des journalistes par les autorités. Mais, le phénomène est plus large et implique à présent un déplacement des menaces physiques sur le terrain des journalistes à celles en ligne passant par du hacking des ordinateurs des professionnel.les de l'information, à leur géolocalisation ou au dévoilement public de certaines de leurs données personnelles². Mais l'enjeu actuellement débattu et largement médiatisé ces dernières années concerne plutôt le discours de haine anti-journalistes et anti-média et le harcèlement en ligne de professionnels. A l'occasion de divers événements et dans des pays très différents, les journalistes témoignent de violences en ligne importantes. Dans leur travail sur les journalistes suédois, Löfren Nillson et Ornebring (2016) montrent de façon extrêmement nette que le tiers des répondant.e.s à leur enquête a expérimenté une augmentation du harcèlement, une diversification de ces formes et a subi des conséquences liées à ces formes d'intimidation. Ils montrent que ces violences peuvent être considérées comme des menaces à l'encontre des médias en tant qu'institution, car les journalistes sont souvent ciblé.e.s pour leur statut professionnel, plutôt que comme représentant une certaine idéologie ou un groupe culturel par exemple. Et pourtant, ils pointent des formes de harcèlement qui touchent frontalement des thématiques liées à l'immigration et à la défense d'une société multiculturelle, ainsi qu'aux questions d'égalité de genre. « Readers' comments, social media, and email, in this sense, represent platforms where different forms of cyberbullying can be used as tactics to force the media to include what is perceived as excluded ideas and groups » (Löfren Nillson \& Ornebring, $2016: 888$ ). Cette pression que l'on pourrait qualifier d'idéologique se couple par ailleurs souvent, 
et c'est le cas pour la France, par exemple, à des attaques qui dépassent la critique du travail journalistique pour développer un positionnement plus radical le plus souvent associé à l'extrême-droite (Mercier et Amigo, 2021).

Mais le harcèlement en ligne ne peut se résumer à des pressions politiques ou idéologiques. Il est aussi très personnalisé. Les journalistes femmes, issu.e.s de groupes dits minorisés ou celles et ceux travaillant sur des thématiques proches des milieux d'extrême-droite sont les plus touché.e.s, et ces pratiques conduisent à des formes de censures populaires, de censures collectives (Waisbord, 2020). Les femmes journalistes sont les plus largement touchées par ces violences (Posetti \& Storm, 2018). De nombreuses études académiques ont vu le jour sur ce sujet ces derniers temps et concernent le cyber-harcèlement et les relations avec les publics (Lewis, Zamith \& Coddington, 2020) les femmes journalistes sportives (Everbach, 2018 ; Antunovic, 2019) et révèlent surtout un mouvement transnational généralisé (Chen et al, 2020). Ces formes de violence proviennent des publics, des sources, et amènent souvent à des témoignages et des dénonciations de la part des femmes journalistes (que l'on pense par exemple, en Belgique, au documentaire récent, \#Sale pute, sur le harcèlement en ligne de femmes médiatiques). Mais ces dénonciations restent encore minoritaires puisque les violences subies amènent souvent les travailleuses de l'information à se retirer des réseaux sociaux, voire même à changer de domaines de spécialisation.

Ces violences publiques devraient par ailleurs être croisées, et ce sera l'objet d'un prochain numéro de la revue Sur le journalisme-About Journalism-Sobre jornalismo, avec les violences organisationnelles, celles qui sont subies par les journalistes au sein même des rédactions. Ici aussi, ces violences sont de diverses natures : hiérarchiques, statutaires, économiques, relationnelles, liées à la carrière, au genre, à l'orientation sexuelle, aux origines, au handicap. Mais, ce numéro actuel sur les violences publiques envers les journalistes et les médias invite, avant d'aller plus loin ou plus proche des conditions quotidiennes de travail, à s'interroger sur le sens de ces violences émises à l'encontre des journalistes. Alors qu'ils et elles se consi- dèrent et sont parfois encore considéré.e.s comme un élément central des régimes démocratiques et du lien entre l'actualité et ce que les publics en connaissent et en perçoivent, les journalistes sont aussi depuis très longtemps l'objet de critiques. Mais les violences en ligne et physiques semblent être devenues un outil politique puissant pour construire et déconstruire les réalités, ou du moins les troubler. Dans un court texte, Reilly, Veneti et Lilleker $\left(2 \mathrm{O}^{3} \mathrm{O}^{3}\right)$ s'inquiètent de l'augmentation des faits de violence auprès des journalistes photographes, caméraman et camerawoman, et journalistes noir.e.s lors de la couverture des manifestations \#BlackLivesMatter et pointent la responsabilité directe de Donald Trump, alors président, de ses attaques récurrentes contre la presse, et du silence des représentant.e.s des autres partis. Toutes les formes de polarisation politique (on en voit l'effet au Brésil, par exemple. Voir Rios \& Bronosky, 2019) engendrent une augmentation des actions violentes envers les médias, comme signe de protestation ; les journalistes devenant alors les cibles désignées, des adversaires (de toute nature parfois). Ce contexte politique entrainant une permissivité accrue de la violence (que l'on pense au recours de l'Etat à la violence, par exemple. Voir Blazquez, 2020) doit aussi être couplé aux contextes économiques de fragilité de nombreux groupes dans la société et d'une représentation de la société largement partagée liée aux risques et aux menaces (environnementales et autres), et empreinte d'incertitude. Dans ce contexte, la pratique journalistique subit la violence, car le monde médiatique non seulement la montre (parfois à outrance), mais la produit aussi. L'invisibilité de certaines thématiques, de certaines personnes, le choix des mots et des expressions pour qualifier certains faits ou certaines réalités, l'hybridité des formes de journalisme et des statuts journalistiques concourent tous à une production de la violence par les médias. Ces derniers triant, excluant, malmenant parfois. Penser les violences publiques envers les médias et les journalistes ne peut se faire sans travailler ce rapport dialectique entre victime et bourreau. En ce sens, le journalisme est violenté, il contribue aussi à provoquer de la violence, mais parfois aussi à conjurer ou juguler certaines formes de violence. Ce numéro thématique de la revue tente, modestement, de travailler certaines de ces pistes.

\section{Notes}

\footnotetext{
${ }^{1 .}$ https://forbiddenstories.org/fr/

${ }^{2}$ Henrichsen, J. R., Betz, M., \& Lisosky, J. M. (2015). Building digital safety for journalism: A survey of selected issues. UNESCO Publishing.

${ }^{3}$ Reilly, P., Veneti, A., \& Lilleker, D. (2020). Violence against journalists is not new, but attacks on those covering\# Black-
}

LivesMatter protests is a bad sign for US press freedom. USApp-American Politics and Policy Blog. http://eprints.lse. ac.uk/105538/1/usappblog_2020_06_12_violence_against_ journalists_is_not_new_but.pdf 


\section{RÉFÉRENCES}

Antunovic, D. (2019). "We wouldn't say it to their faces": online harassment, women sports journalists, and feminism. Feminist Media Studies, 19(3), 428-442.

Badran, M. (2017). Violence against journalists: suppressing media freedom. In Digital Transformation in Journalism and News Media (pp. 417-427). Springer, Cham.

Benítez, J. L. (2017). Violence against journalists in the Northern Triangle of Central America. Media Asia, 44(1), 61-65.

Blazquez, A. (2020). Désirs de justice, attente de l'État et recours à la violence. État et société politique, 105.

Brambila, J. A., \& Hughes, S. (2019). Violence against journalists. The international encyclopedia of journalism studies, 1-9.

Chen, G. M., Pain, P., Chen, V. Y., Mekelburg, M., Springer, N., \& Troger, F. (2020). 'You really have to have a thick skin': A cross-cultural perspective on how online harassment influences female journalists. Journalism, 21(7), 877-895.

Everbach, T. (2018). "I realized it was about them... not me": Women sports journalists and harassment. In Mediating misogyny (pp. 131-149). Palgrave Macmillan, Cham.

Fang, K. (2017). "Guard against fire, theft, and journalists": the public against the press in China. Media Asia, 44(1), 55-60

Frère, M. S. (2015). Journaliste en Afrique: métier à risque et risques pour le métier. Du risque en Afrique. Terrains et perspectives, Paris, Karthala, 132-153.

González de Bustamante, C., \& Relly, J. E. (2016). The practice and study of journalism in zones of violence in Latin America: Mexico as a case study. Journal of Applied Journalism \& Media Studies, 5(1), 51-69.

González de Bustamante, C., \& Relly, J. E. (2016b). Professionalism under threat of violence: Journalism, reflexivity, and the potential for collective professional autonomy in northern Mexico. Journalism Studies, 17(6), 684-702.

Harb, Z. (2019). Challenges facing Arab journalism, freedom, safety and economic security.Journalism, 20(1), 110-113.

Jamil, S. (2018). Freedom of expression and threats to journalists' safety: an analysis of conflict reporting in journalism education in Pakistan. Journalism, 6(2).

Joux, A. (2019). Des journalistes pas comme les autres. Les Cahiers du numérique, 15(3), 29-52.

Konow-Lund, M., \& Olsson, E. K. (2020). Cross-Border Investigative Collaboration on the Surviving Stories: The Forbidden Stories. In Critical Incidents in Journalism (pp. 191202). Routledge.

Lewis, S. C., Zamith, R., \& Coddington, M. (2020). Online harassment and its implications for the journalist-audience relationship. Digital Journalism, 8(8), 1047-1067.
Louback, A. C. S. (2018). Jornalistas negras no Rio de Janeiro: trajetórias de vida e narrativas de resistência diante do racismo. Dissertação de Mestrado. Rio de Janeiro : CEFET-RJ. Disponível em: http://dippg.cefet-rj.br/pprer/attachments/article/81/102_Andreia\%20Coutinho\%20da\%20 Silva\%20Louback.pdf

Mercier, A., \& Amigo, L. (2021). Tweets injurieux et haineux contre les journalistes et les «merdias». Mots. Les langages du politique, (125), 73-91.

Nerone, J. (2008). Violence against Journalists. The International Encyclopedia of Communication.

Palmer, L. (2019). The fixers: Local news workers and the underground labor of international reporting. Oxford University Press.

Posetti, J., \& Storm, H. (2018). Violence Against Women Journalists-Online and Offline. Setting the Gender Agenda for Communication Policy: New Proposals from the Global Alliance on Media and Gender, 75-86.

Ramos, D. O. \& Saad, E. Violência digital contra jornalistas: o caso das eleições presidenciais de 2018. Actes du XXIX Encontro Anual da Compós. Campo Grande : UFMS/Compós. Disponível em: http://www.compos.org. br/biblioteca/trabalhos_arquivo_GF08O9WXJ33HGJ5Q GZ69_30_8278_13_02_2020_18_52_07.pdf

Relly, J. E., \& González de Bustamante, C. (2017). Global and domestic networks advancing prospects for institutional and social change: the collective action response to violence against journalists. Journalism \& Communication Monographs, 19(2), 84-152.

Rios, A., \& Bronosky, M. (2019). Violência contra jornalistas, ameaça à sociedade. Mosaico, 11(17), 86-103.

Sebbah, B., Loubère, L., Souillard, N., Thiong-Kay, L., \& Smyrnaios, N. (2018). Les Gilets jaunes se font une place dans les médias et l'agenda politique. Rapport de recherche Lerass.

Sreberny, A. (2014). Violence against women journalists. Media and Gender: A Scholarly Alliance for the Global Alliance on Media and Gender, 35-39.

Tumber, H. (2006). The fear of living dangerously: Journalists who report on conflict. International Relations, 20(4), 439-451.

Torsner, S. (2017). Measuring journalism safety. Methodological challenges.

Waisbord, S. (2020). Mob censorship: Online harassment of US journalists in times of digital hate and populism. Digital Journalism, 8(8), 1030-1046. 